\title{
Implementation of 1-Bit Random Access Memory Cell in All-Optical Domain with Non-linear Material
}

\author{
Samir Sahu ${ }^{1, *}$, Radha Raman Pal ${ }^{1}$, Shantanu Dhar ${ }^{2}$ \\ ${ }^{1}$ Department of Physics and Technophysics, Vidyasagar University, Midnapore, 721102, India \\ ${ }^{2}$ Department of Physics, Jhargram Raj College, Jhargram, 721507, India
}

\begin{abstract}
This paper demonstrates an all-optical 1-bit Random Access Memory (RAM) with massive use of nonlinear material. All-optical switching mechanism is exploiting here to realize the all-optical 1-bit RAM. The all-optical switch by a composite slab of linear medium (LM) and non-linear medium (NLM) is the building block of our proposed 1-bit RAM circuit. An all-optical clocked D flip flop is the main storing element of the RAM. These circuits are simple and all-optical in nature. It can also gear up to the highest capability of optical performance in high-speed all-optical data storing, computing and communicating system.
\end{abstract}

Keywords Nonlinear Material, All-Optical Switch, All-Optical D Flip-Flop, 1-Bit Read/Write Memory Cell, All-Optical RAM

\section{Introduction}

To cope with the explosive growth of the internet traffic, the expected necessity for parallel, ultrahigh speed, ultrahigh bandwidth, no crosstalk, low loss information processing[1] has provided remarkable impetus for understanding of all-optical devices and circuits[1-3]. Last few years have witnessed an enhanced weight in optical communication[3-4] and computing[5] due to some important advancement, namely, the development of laser, optical fiber and novel materials that exhibit high nonlinearities[1, 5-12]. These developments of optics offer tremendous scope to overcome the obstacle to implement various arithmetic[2, 7-8], logic[9-14, 18-20], algebraic[15] and image operations[16] in all-optical domain successfully. The advent of non-linear optical material based all-optical switching mechanism[1, $5-12,18,25]$ has opened exciting new possibilities for improvement of several new all-optical components of terahertz all-optical computer, our dream goal.

This promising technique of all-optical switching can show its potential in combinational logic circuits $[2,7-8,17]$ as well as sequential logic circuits[9-12, 18]. For examples, all-optical S-R, S-R with clock, D, J-K and J-K master-slave type flip-flops using non-linear material[9-12, 18-20], all optical register[11, 22-23], all optical counter[12] have been reported. Various all-optical memories have also been pro posed[22-24]. They are not as fast as to fulfil the demand of

* Corresponding author:

tosamirsahu@gmail.com (Samir Sahu)

Published online at http://journal.sapub.org/optics

Copyright (C) 2011 Scientific \& Academic Publishing. All Rights Reserved now-a-days. To gear up the performance speeds (storing speed and retrieving speed) we implement an all-optical 1-bit random access memory[21] which is constructed by the clocked D flip flop[9,11].This circuit can raise to a higher bit other types of RAM. As this 1-bit RAM is purely all-optical in nature, it is very simple as well as very fast. Also the scheme has capacity of cascading. These circuits are key elements for the implementation of a high-speed, all-optical data processing device, which has the potential to outperform its electronic equivalent and constitute a possible new product for next generation computation[1,5] and communication[3-4] to scale their capacity to the traffic demand.

\section{Ultra Fast All-optical Switching behaviour of Nonlinear Material and their Application in All-Optical NOT Gate as well as AND Gate}

The phenomenon photorefractivity $[6,8-12]$ of some nonlinear optical material is used in nonlinear all-optical intensity switching mechanism. The refractive index[1, 6 , 8-12, 25-27] of some nonlinear materials (NLM) such as carbon disulfide, pure silica, potassium dihydrophosphate $\left(\mathrm{KH}_{2} \mathrm{PO}_{4}\right)$, (KDP) crystal etc. varies linearly with the intensity of the light incident on it. The refractive index (n) of such isotropic dielectric non-crystalline media can be put into an equation as in (1). Here $\mathrm{n}_{0}$ is the linear term, $\mathrm{n}_{1}$ is the nonlinear correction term and I is the intensity of the incident light beam on the material.

$$
\mathrm{n}=\mathrm{n}_{0}+\mathrm{n}_{1} \mathrm{I}
$$




\subsection{Ultra Fast All-Optical Switching Behavior of Nonlinear Material}

We can implement the switching mechanism with such nonlinear material by taking an interface between two media of which one is a linear material (LM), whose refractive index $\mathrm{n}_{0}$ is independent of the intensity of light and the other is aforesaid NLM. A laser beam, highly intense polarized light, preferably pulse laser of intensity $\mathrm{I}_{1}$, is allowed to incident on the interface from linear to nonlinear part in a particular direction XO (incidence angle $\theta_{1}$ ) as depicted in Figure 1. The refracted beam from the NLM follows the path OZ. But when another higher intense laser beam of intensity $\mathrm{I}_{2}\left(\mathrm{I}_{2}>\mathrm{I}_{1}\right)$ is made to incident along XO, after refraction from the NLM the light passes through OY direction (angle of refraction $\theta_{2}$ ). The deviation of refractive angle for different incident light intensity $I_{1}$ and $I_{2}$ is $<Z O Y=\Delta \theta_{2}$. Thus the combination of LM and NLM may act nicely as a directional all-optical switch. This is the unit block of our proposed 1-bit random access memory cell.

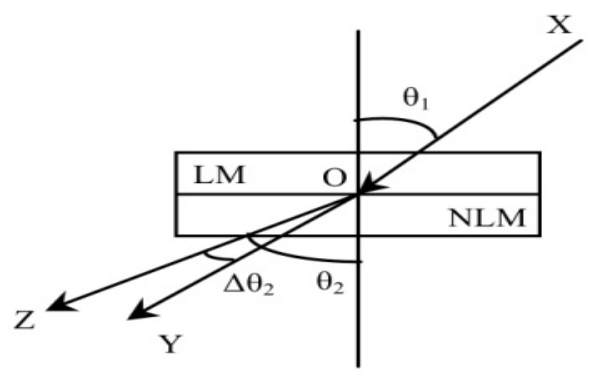

Figure 1. Intensity switching of optical nonlinear material

In the expression of refractive index in (1) $\mathrm{n}_{0}$ is linear term and $\mathrm{n}_{1}$ is the nonlinear correction term. For carbon disulfide [8, 10-12, 25] $\left(\mathrm{CS}_{2}\right) \mathrm{n}_{0}=1.63, \mathrm{n}_{1}=514 \times 10^{-20} \mathrm{~m}^{2} / \mathrm{W}$. and for fused silicon dioxide $[8,10-12,25]\left(\mathrm{SiO}_{2}\right) \mathrm{n}_{0}=1.458, \mathrm{n}_{1}=$ $2.7 \times 10^{-20} \mathrm{~m}^{2} / \mathrm{W}$. If we use $\mathrm{CS}_{2}$ and $\mathrm{SiO}_{2}$ as nonlinear materials and the pulse laser of intensity $\mathrm{I}=2 \times 10^{18} \mathrm{~W} / \mathrm{m}^{2}$ as a source, we can estimate the deviations of light in two cases as given in Table 1.

The logic gates[7-12, 18] are implemented in optics using NLM by taking the presence of light signal as 1 and the absence of it as 0 . The implementation of such logic gates can be done by using some femtosecond laser pulses and 1 -mm-thick KDP crystal at the pick intensity of $0.6 \mathrm{TW} / \mathrm{cm}^{2}$ and duration of $60 \mathrm{fs}[8,10-12,26]$. M. Choi et al. show that a single-layer terahertz metamaterial[27] has a peak refractive index of 38.6 while maintaining low losses. It is a broadband, extremely high index of refraction going beyond the limit that is attainable with naturally existing substances, lead sulphide, strontium titanate[27].

\subsection{All Optical NOT Gate}

To implement an all optical NOT gate using non-linear material a constant intensity pulse laser source (CILS) is used as shown in Figure 2. It is also called probe beam. Here $\mathrm{P}_{1}$ is taken as input beam. A detector is placed at $\mathrm{P}_{2}$ will detect the output beam after refraction. If $\mathrm{P}_{1}$ is absent, the light will follow a path $\mathrm{OP}_{2}$ and will be detected by the detector due to presence of CILS. But if $\mathrm{P}_{1}$ is present, after refraction, the light will follow a path other than $\mathrm{OP}_{2}$, may be $\mathrm{OP}_{3}$, and the detector will not detect any light signal. Thus the system acts as optical NOT gate.

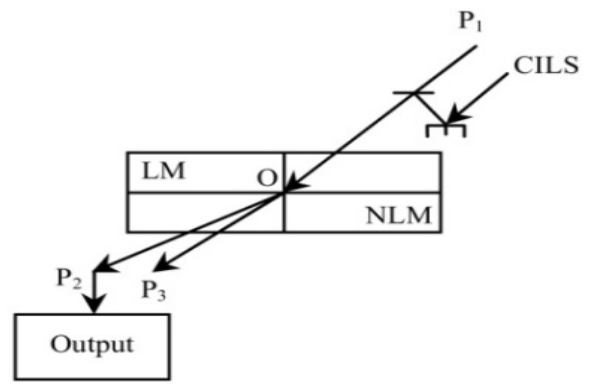

Figure 2. All-optical NOT gate

\subsection{All Optical AND Gate}

The all-optical AND gate using two inputs and three inputs are shown in Figure 3. The two inputs all-optical AND gate using NLM is shown in Figure 3(a). Here $R_{1}$ and $R_{2}$ are two input channels. A detector placed at $\mathrm{R}_{4}$ gives the output. Now when both the channels carry light signal, the light beam after refraction will detected by the detector at $\mathrm{R}_{4}$, unless not.

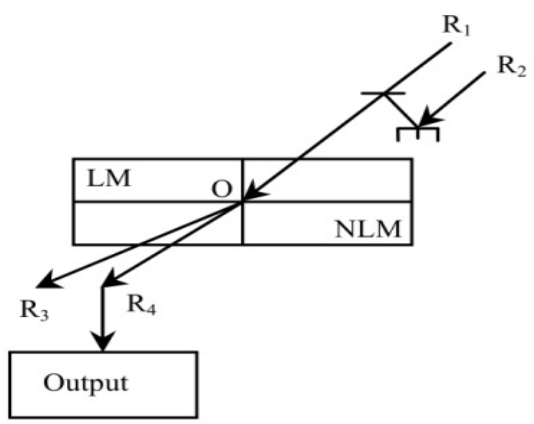

(a)

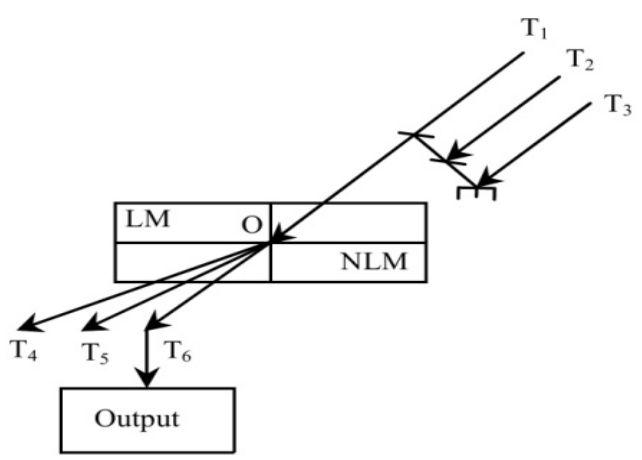

(b)

Figure 3. All-optical AND gate using NLM. (a) two-input AND gate. (b) three-input AND gate

The three inputs all-optical AND gate using NLM is shown in Figure $3(b)$. Here $T_{1}, T_{2}$ and $T_{3}$ are three input channels. A detector placed at $\mathrm{T}_{6}$ gives the output. Now when all the channels carry light signal, the light beam after refraction will detected by the detector at $\mathrm{T}_{6}$, unless not. 
Table 1. Estimation of the Deviation of Pulsed Laser Light when Passing Through Carbon Disulfide $\left(\mathrm{CS}_{2}\right)$ and Silicon Dioxide $\left(\mathrm{SiO}_{2}\right)$

\begin{tabular}{cccccc}
\hline \hline Material & $\begin{array}{c}\text { Angle of } \\
\text { Incidence }\left(\theta_{1}\right)\end{array}$ & $\begin{array}{c}\text { Incident light } \\
\text { intensity }\end{array}$ & $\begin{array}{c}\mathrm{n} \\
\left(=\mathrm{n}_{0}+\mathrm{n}_{1} \mathrm{I}\right)\end{array}$ & $\begin{array}{c}\text { Angle of } \\
\text { refraction }\left(\theta_{2}\right)\end{array}$ & $\begin{array}{c}\text { Deviation } \\
\left(\Delta \theta_{2}=\theta_{2}^{\prime}-\theta_{2}^{\prime \prime}\right)\end{array}$ \\
\hline $\begin{array}{c}\text { carbon disul- } \\
\text { fide }\left(\mathrm{CS}_{2}\right)\end{array}$ & $45 \mathrm{deg}$ & $\mathrm{I}=2 \times 10^{18} \mathrm{~W} / \mathrm{m}^{2}$ & 11.91 & $3.404 \mathrm{deg}=\theta_{2}^{\prime}$ & $1.578 \mathrm{deg}$ \\
$\begin{array}{c}45 \mathrm{deg} \\
\text { silicon }\end{array}$ & $2 \mathrm{I}$ & 22.19 & $1.827 \mathrm{deg}=\theta_{2}^{\prime \prime}$ & \\
di-oxide $\left(\mathrm{SiO}_{2}\right)$ & $45 \mathrm{deg}$ & $\mathrm{I}=2 \times 10^{18} \mathrm{~W} / \mathrm{m}^{2}$ & 1.512 & $27.883 \mathrm{deg}=\theta_{2}^{\prime}$ & \\
\hline \hline
\end{tabular}

\section{All-optical 1-bit Random Access Memory Cell}

We now describe the all-optical circuit of 1-bit random access memory (RAM) cell as shown in Figure 4 . Here $D_{i}$ is the Data Input of the 1-bit RAM and $\mathrm{D}_{0}$ is the Data Output of the cell. There are two other inputs namely Address Select input $\left(\mathrm{A}_{\mathrm{n}}\right)$ and $\mathrm{Read} /$ Write Control input $(\mathrm{R} / \bar{W})$. The main storing component of this RAM cell is the clocked D flip-flop $[9,11]$ shown in the dash box as in Figure 4. The truth table is depicted in Table 2. Except the D flip-flop, here we use one all-optical NOT gate (NG6) and two AND gates (AG5 and AG6). AG5 is a two-input AND gate while AG6 is a three-input AND gate. All the gates are based on optical nonlinear material as the basic building block of our proposed RAM cell. The $\mathrm{R} / \bar{W}$ input is complemented at the $\mathrm{D}_{10}$ terminal through NG6 due to the presence of probe beam CILS6. Now AG5 has $A_{n}$ and $D_{10}$ as two inputs and $E_{11}$ as output. Again Data Input $\left(D_{i}\right)$ of the RAM cell is fed to $D$ input of the $\mathrm{D}$ flip-flop and $\mathrm{E}_{11}$ is fed as clock (CLK) of the $\mathrm{D}$ flip-flop. The flip-flop has outputs $\mathrm{Q}$ and $\bar{Q}$, complement to each other. $\mathrm{A}_{\mathrm{n}}, \mathrm{R} / \bar{W}$ and $\mathrm{Q}$ are the three inputs of the AND gate AG6. The output $\left(\mathrm{F}_{12}\right)$ of AG6 is the final output $\left(\mathrm{D}_{0}\right)$ of the proposed all-optical 1-bit Read/Write memory cell.

Table 2. Truth Table of Clocked D Flip-Flop

\begin{tabular}{ccccc}
\hline \hline \multicolumn{2}{c}{ Inputs } & & \multicolumn{2}{c}{ Outputs } \\
\cline { 1 - 2 } CLK & $\mathrm{D}$ & & $\mathrm{Q}_{\mathrm{n}+1}$ & $\bar{Q}_{\mathrm{n}+1}$ \\
\hline 0 & $\mathrm{~d}$ & & $\mathrm{Q}_{\mathrm{n}}$ & $\bar{Q}_{\mathrm{n}}$ \\
1 & 0 & & 0 & 1 \\
1 & 1 & 1 & 0 \\
\hline \hline & &
\end{tabular}

Let us discuss the operation of the circuit to form the function table of the 1-bit RAM cell.

(1) First we take $A_{n}=0$, i.e. $A_{n}$ carries no light signal. As AG5 and AG6 are AND gates, $\mathrm{E}_{11}\left(=\mathrm{CLK}=\underline{0)}\right.$ and $\mathrm{F}_{12}\left(=\mathrm{D}_{0}\right.$ $=0)$ remain dark irrespective of the inputs $\mathrm{R} / \bar{W}$ and $\mathrm{D}_{\mathrm{i}}$. Now CLK of the D flip-flop is absent which preserved the previous state $\left(Q_{n+1}=Q_{n}\right)$. So as long as $A_{n}=0$, the input and output activities are blocked, and the cell is in the hold mode where its stored data is protected.

(2) If Address Select input bears photon $\left(A_{n}=1\right)$, the cell is enabled for writing and reading operation. There are two possibilities.

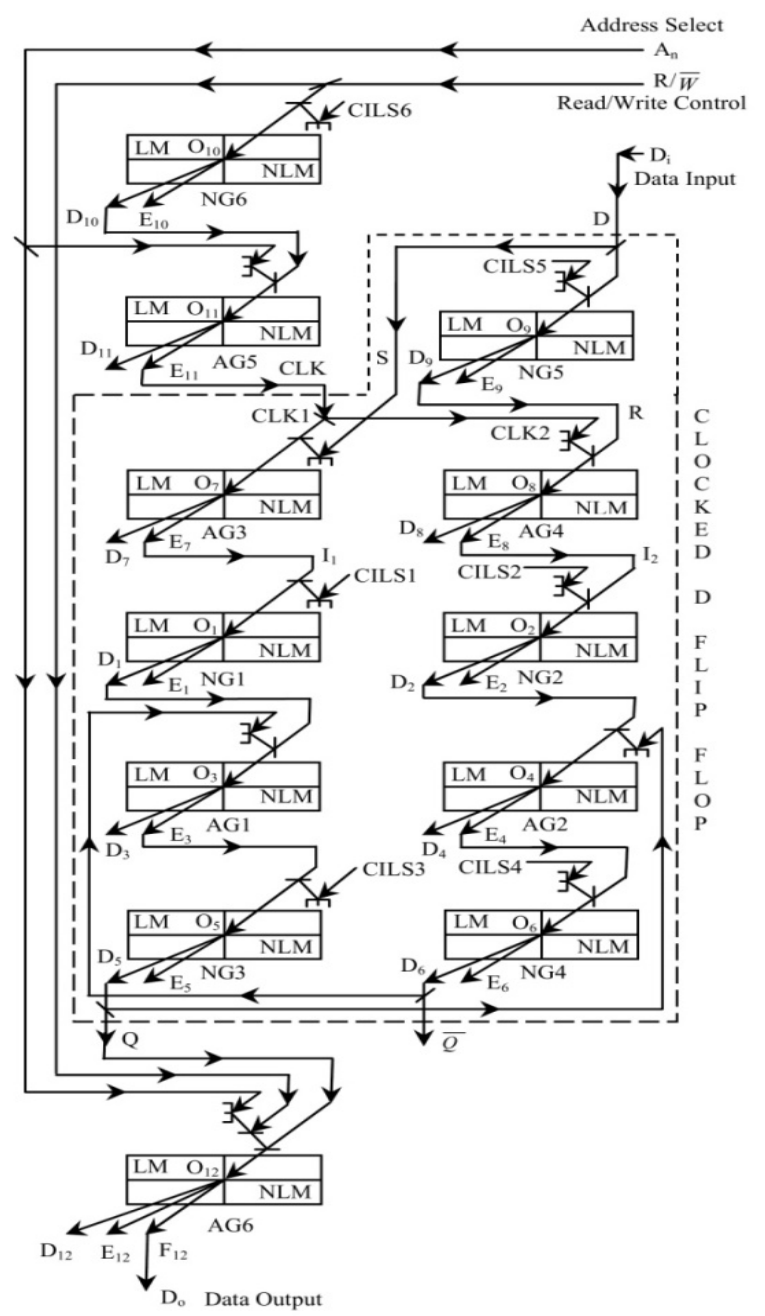

Figure 4. All optical random-access memory cell using NLM as switch

i) The first possibility, $\mathrm{R} / \bar{W}$ is equal to 0 . Then the output $\mathrm{D}_{0}\left(=\mathrm{F}_{12}\right.$, output of AG6) becomes 0 independent of Q. i.e. the output of the cell is detached from Q. One cannot read from the memory cell in this state. But light will follow the path $\mathrm{O}_{11} \mathrm{E}_{11}$ when passing through AG5 as both the input beams $\left(A_{n}=D_{10}=1\right)$ have light. Now the clock of the $D$ flip-flop becomes at logical ' 1 ' state. So the D flip-flop stores 0 if $\mathrm{D}_{\mathrm{i}}=\mathrm{D}=0$ and writes 1 if $\mathrm{D}_{\mathrm{i}}=\mathrm{D}=1$ according to Table 2. Therefore, if $A_{n}=1$ and $\mathrm{R} / \bar{W}=0$, writing (storing) operation of single bit data $\left(\mathrm{D}_{\mathrm{i}}\right)$ is done into the memory cell. 
Table 3. Function Table of All-Optical 1-Bit Read/Write Memory Cell

\begin{tabular}{|c|c|c|c|c|c|c|}
\hline \multicolumn{3}{|c|}{ Inputs } & \multicolumn{3}{|c|}{ Outputs } & \multirow[t]{2}{*}{ Mode } \\
\hline$A_{n}$ & $\mathrm{R} / \bar{W}$ & $\mathrm{D}_{\mathrm{i}}=\mathrm{D}$ & CLK & $\mathrm{Q}_{\mathrm{n}+1}$ & $\mathrm{D}_{0}$ & \\
\hline 0 & d & $\mathrm{d}$ & 0 & $\mathrm{Q}_{\mathrm{n}}$ & 0 & Hold \\
\hline 1 & 0 & 0 & 1 & 0 & 0 & Write 0 into memory \\
\hline 1 & 1 & $\mathrm{~d}$ & 0 & 0 & 0 & Read, $\mathrm{D}_{0}=$ stored $\mathrm{D}_{\mathrm{i}}$ bit \\
\hline 1 & 0 & 1 & 1 & 1 & 0 & Write 1 into memory \\
\hline 1 & 1 & $\mathrm{~d}$ & 0 & 1 & 1 & Read, $\mathrm{D}_{0}=$ stored $\mathrm{D}_{\mathrm{i}}$ bit \\
\hline
\end{tabular}

$\mathrm{d}=$ whatever may be the input

ii) In the last possibility we consider $\mathrm{R} / \bar{W}$ as 1 . There will be no light at $\mathrm{E}_{11}$ terminal as $\mathrm{D}_{10}=0$. Again the clock of the D flip-flop is absent. The flip-flop remain in its former output state ignoring $\mathrm{D}\left(=\mathrm{D}_{\mathrm{i}}\right)$ input. In the present situation none can write into the memory cell. But for AG6, as two inputs $\left(\mathrm{A}_{\mathrm{n}}=\mathrm{R} / \bar{W}=1\right)$ are at logically high state, the output follows the third input $\mathrm{Q}$. That means $\mathrm{Q}$ comes out at $\mathrm{D}_{0}$ through $\mathrm{O}_{12} \mathrm{~F}_{12}$ direction. $\mathrm{Q}$ is nothing but the stored $\mathrm{D}_{\mathrm{i}}$ bit. So we can conclude that stored digital data will be retrieved from 1-bit read/write memory cell when both the inputs $A_{n}$ and $\mathrm{R} / \bar{W}$ transmit light.

The function table is given in Table 3. All-optical 1-bit RAM cell can store digital optical data and retrieve it as desired. In our scheme we use all optical AND and NOT gate in designing the all optical 1-bit RAM cell. In our design the light beam which is fed back is coming from the output of a NOT gate. Again the concept used here to design the all optical NOT gate has an advantage. Whenever the output of a NOT gate is assumed to be at ' 1 ' state, the source of that ' 1 ', state is a constant intensity pulse laser source (CILS) used as probe beam. So in each feedback arrangement described in our scheme similar intense light beam is fed back. In this way the reduction of intensity by using beam splitter will not affect the non-linear response of the device. The light sources are so chosen that each input beam intensity is in the range of intensity which is detected as ' 1 ' by the detector after refraction. The source of the final output $\left(\mathrm{D}_{0}\right)$ of the RAM cell is CILS3. As long as the power is on, the stored data bit is protected and the data retrieval is possible. Therefore the proposed all-optical RAM cell can be used as volatile memory cell.

\section{Conclusions}

The proposed technique of all optical implementation of all optical 1-bit RAM cell is very fast (above THz)[8-12, 26-27] as it is fully all-optical. It is volatile in nature. The storing and reading time of this memory cell will be in fs. The light signals which are severally used and the feedback light signals from the outputs are made by mirrors and beam splitters to make the circuits simple. Another important feature is that other higher order all-optical temporary data storage memories may be developed by cascading the cell.
Other different types of memories can be implemented from this read/write memory cell. Proper findings of non-linear material[25-27] may be a significant issue here. Essentially inputs and constant intensity light source should be chosen properly to run the system accurately. The clock pulse signal should also be selected suitably.

\section{ACKNOWLEDGEMENTS}

The authors want to acknowledge Professor Sourangshu Mukhopadhyay of the University of Burdwan for his valuable suggestions.

\section{REFERENCES}

[1] D. Cotter, R. J. Manning, K. J. Blow, A. D. Ellis, A. E. Kelly, D. Nesset, I. D. Phillips, A. J. Poustie and D. C. Rogers, "Nonlinear Optics for High-Speed Digital Information Processing," SCIENCE, 286, 1523-1528, 1999

[2] Sukhdev Roy, Mohit Prasad, Juraj Topolancik and Frank Vollmer, "All-Optical Arithmetic and Combinatorial Logic Circuits with High-Q Bacteriorhodopsin Coated Microcavities," Cornel University Library, 1-39. Available: http://www.arxiv.org/pdf/1001.0244

[3] P. Huang, F. Luo, M. Cao, Y. Yang and Y. Feng, "Optical interconnecting and switching network system of 5 to 10 -Gbps bandwidth for parallel computing," Proc. SPIE. 5281, 559-566, 2004

[4] D. Hillerkuss et al., "26 Tbit s-1 line-rate super-channel transmission utilizing all-optical fast Fourier transform processing," Nature Photonics. 5, 364-373, 2011

[5] J. Zhang and H. Xu, "Optical computation based on nonlinear total reflectional optical switch at the interface," Pramana. J. Phys. 72(3), 547-554, 2009

[6] D. Arivouli, "Fundamentals of optical nonlinear materials," Pramana. 57(5-6) 871-883, 2001

[7] D. K. Gayen and J. N. Roy, "All-optical arithmetic unit with the help of terahertz- optical-asymmetric- demultiplexer-based tree architecture," Appl. Opt. 47(7), 933-943, 2008

[8] S. Sahu, R. R. Pal and S. Dhar, "A Novel Method of Implementing Nonlinear Material Based All-Optical Binary Half 
Subtractor and Full Subtractor System," J. of Electron Devices. 10, 493-498, 2011

[9] S. Dhar and S. Sahu, "All-optical implementation of S-R, clocked S-R and D flip-flops using nonlinear material," Opt. Eng. 47(6), 065401-1-6, 2008

[10] S. Sahu and S. Dhar, "Implementation of clocked J-K, T and J-K Master Slave flip-flops with nonlinear material in All-optical Domain,” Opt. Eng. 48(7), 075401-1-7, 2009

[11] S. Sahu, R. R. Pal and S. Dhar, "TeraHertz All-Optical Binary Register using D flip-flop with Non-linear Material: A Proposal" J. of Electron Devices. 11, 588-595, 2011

[12] S. Sahu, R. R. Pal and S. Dhar, "All-Optical Binary Counter by using T flip-flop: An Implementation" International Journal of Engineering, Science and Technology. 3(10), 7799-7807, 2011

[13] A. Meiri and Z. Zalevsky, "Nano electro-optical modulator and all-optical logic gate on a silicon chip," J. Nanophoton. 5 , 051811, 2011

[14] Q. Xu and M. Lipson, "All-optical logic based on silicon micro-ring Resonators," Opt. Exp. 15(3), 924-929, 2007

[15] A. Andreoni, M. Bondani, M.A.C. Potenza, Y.N. Denisyuk and E. Puddu, "Boolean algebra operations performed on optical bits by the generation of holographic fields through second-order nonlinear interactions," Rev. Sci. Instrumn. 72, 2525-2531, 2001

[16] A. Yang, Z. Liu, B. Wang, Y. Zhang and S. Liu, "Optical stream-cipher-like system for image encryption based on Michelson interferometer," Opt. Exp. 19(3), 2634-2642, 2011

[17] P. S. Guilfoyle and W. J. Wiley, "Combinational logic based digital optical computing architecture," Appl. Opt. 27, 1661-1673, 1988
[18] N. Pahari and S. Mukhopadhyay, "An all optical R-S flip-flop by optical non-linear material," J. of Opts. 34 (3), 108114, 2005

[19] A. K. Datta and S. Munshi, "Optical implementation of flip-flops using single-LCD panel," Opt. \& Laser Tech. 39(9), 2321-2329, 2007

[20] T. Chattopadhyay, "All-optical clocked delay flip-flop using a single terahertz optical asymmetric demultiplexer-based switch: a theoretical study," Appl. Opt. 49, 5226-5235, 2010.

[21] R. P. Jain, Modern Digital Electronics, $3^{\text {rd }}$ ed., Tata MaGraw-Hill India, New Delhi. India: 2007

[22] Tamer A. Moniem, Nabil Abd Rabou, and E. M. Saad, "Parallel-shift register and binary multiplier using optical hardware components," Opt. Eng. 47(3), 035201 (2008)

[23] E. Lazzeri, G. Berrettini, G. Meloni, A. Bogoni and L. Poti, "All-Optical-Bits Shift Register Exploiting a Ring Buffer Based on Semiconductor Optical Amplifier," Phot. Tech. Lett. IEEE. 23(1), 45-47, 2011

[24] G. Berrettini, L. Potì and A. Bogoni, "Optical Dynamic RAM for All-Optical Digital Processing," Photonics Technology Letters, IEEE. 23(11), 685-687, 2011

[25] D Samanta, S. Mukhopadhyay, "A method of maintaining the intensity level of a polarization encoded light signal," Journal of Physical Science. 11, 87-91, 2007

[26] S. Mironov. V. Lozhkarev, V. Ginzburg and E. Khazanov, "High-efficiency second-hermonic generation of superintense ultrashort laser pulses," Applied Optics. 48, 2051-2057, 2009

[27] M. Choi, S. H. Lee, Y. Kim, S. B. Kang, J. Shin, M. H. Kwak, K.Y. Kang, Y. H. Lee, N. Park and B. Min, "A terahertz metamaterial with unnaturally high refractive index," Nature. 470, 369-373, 2011 